\title{
Radiative impacts of cloud heterogeneity and overlap in an atmospheric General Circulation Model
}

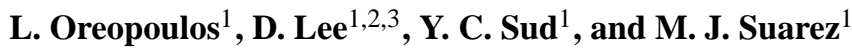 \\ ${ }^{1}$ NASA Goddard Space Flight Center, Greenbelt, MD, USA \\ ${ }^{2}$ University Space Research Association, Columbia, MD, USA \\ ${ }^{3}$ Seoul National University, Seoul, South Korea
}

Correspondence to: L. Oreopoulos (lazaros.oreopoulos@nasa.gov)

Received: 16 April 2012 - Published in Atmos. Chem. Phys. Discuss.: 12 May 2012

Revised: 30 August 2012 - Accepted: 20 September 2012 - Published: 4 October 2012

\begin{abstract}
The radiative impacts of horizontal heterogeneity of layer cloud condensate, and vertical overlap of both condensate and cloud fraction are examined with the aid of a new radiation package operating in the GEOS-5 Atmospheric General Circulation Model. The impacts are examined in terms of diagnostic top-of-the atmosphere shortwave (SW) and longwave (LW) cloud radiative effect (CRE) calculations for a range of assumptions and overlap parameter specifications. The investigation is conducted for two distinct cloud schemes, one that comes with the standard GEOS-5 distribution, and another used experimentally for its enhanced cloud microphysical capabilities. Both schemes are coupled to a cloud generator allowing arbitrary cloud overlap specification. Results show that cloud overlap radiative impacts are significantly stronger in the operational cloud scheme where a change of cloud fraction overlap from maximum-random to generalized results in global changes of SW and LW CRE of $\sim 4 \mathrm{Wm}^{-2}$, and zonal changes of up to $\sim 10 \mathrm{Wm}^{-2}$. This is an outcome of fewer occurrences (compared to the other scheme) of large layer cloud fractions and fewer multi-layer situations where large numbers of atmospheric layers are simultaneously cloudy, both conditions that make overlap details more important. The impact of the specifics of condensate distribution overlap on CRE is much weaker. Once generalized overlap is adopted, both cloud schemes are only modestly sensitive to the exact values of the overlap parameters. When one of the CRE components is overestimated and the other underestimated, both cannot be driven simoultaneously towards observed values by adjustments to cloud condensate heterogeneity and overlap specifications alone.
\end{abstract}

\section{Introduction}

With recent computationally efficient approaches to treat cloud-radiation interactions, there are now fewer reasons to retain the simplistic cloud descriptions that have persisted in General Circulation Models (GCMs) for the last three decades. Clouds do no longer have to be treated by the radiation schemes of these models as homogeneous slabs within large areas $\mathrm{O}\left(10^{4} \mathrm{~km}^{2}\right)$, with fractional coverages and optical depths or water paths adjusted (Tiedtke, 1996; Sud and Walker, 1999; Molod et al., 2012) to rectify the biases that would otherwise plague modeled radiation fields. While capturing the radiative effects of full-blown 3-D cloud heterogeneity may still be elusive, the representation of in-cloud horizontal heterogeneity of cloud condensate and two-point statistics of vertical correlations of condensate and cloud fraction within a one-dimensional radiative transfer framework is now feasible. As a matter of fact, the current work is one more study that amply demonstrates the viability of such an undertaking.

The main development that makes more complex cloud descriptions possible is the introduction of methods that perform radiative transfer in the cloudy portions of GCM gridcolumns in a stochastic manner (Pincus et al., 2003). The more complex cloud descriptions come from cloud generators producing horizontal and vertical cloud variability according to rules that are relatively easy to implement. The cloud fields from the generators can then be coupled with the stochastically operating radiative transfer schemes that receive as input atmospheric subcolumns for which cloud fraction is unity and condensate is horizontally invariable 
whenever a layer is cloudy. With the radiative transfer simplified, the sensitivity of the radiation budget to a variety of specifications transforming a gridcolumn's cloud profile to a cloud field consisting of several subcolumns can be easily examined. What should ultimately be investigated is whether the effects of cloud complexity on the transfer of solar and thermal infrared radiation matter for the GCM's climate. Such a study on the full impacts of interactions and feedbacks of the altered radiation fields with the multitude of the GCM's dynamical and physical processes is left for the future. Here, we simply focus on diagnosing the possible range of radiative impacts of enhanced cloud complexity, an approach akin to that of Shonk and Hogan (2010).

In the following we will present the tools, assumptions, and experimental setup that allow us to examine the degree to which cloud complexity changes the cloud radiative impact (Sects. 2, 3, and 4). The availability of two cloud schemes in our GCM combined with our analysis approach provides the opportunity to investigate whether identical assumptions about cloud complexity imposed on different original cloud fields can yield notably distinct radiative impacts (Sect. 5) and the reasons behind the dissimilar behaviours (Sect. 6).

\section{Implementation of RRTMG into GEOS-5}

The effects of cloud overlap (fraction and condensate) on the radiative fluxes can be captured best with radiation codes equipped with flexibility in the representation of such overlap. This (along with improved representation of gaseous absorption) was one of the primary motivations for the implementation into the GEOS-5 Atmospheric General Circulation Model (AGCM, Rienecker et al., 2010; Molod et al., 2012) of the RRTMG radiation package (Clough et al., 2005), a faster incarnation of the RRTM codes (Mlawer et al., 1997; Iacono et al., 2008) designed specifically for large scale models, and consisting of solar and thermal infrared components. Both components can be run in so-called Monte Carlo Independent Column Approximation (McICA) mode (Pincus et al., 2003). RRTMG with McICA has been implemented succesfully into ECMWF's Integrated Forecasting System (Morcrette et al., 2008) and several other large scale models. Within the McICA framework, when the radiation code is employed on a number of atmospheric (sub)columns, full spectral integration over each column is replaced by stochastic (Monte Carlo) integration. A simplified mathematical expression of this process can be written as follows:

$\bar{F}=\frac{1}{N} \sum_{n=1}^{N} F_{n}=\frac{1}{N} \sum_{n=1}^{N} \sum_{k=1}^{K} f_{n, k} \approx \sum_{k=1}^{K} f_{n_{k}, k}$

The uppercase symbols of Eq. (1) represent broadband fluxes, while the lowercase letters represent pseudomonochromatic fluxes per the correlated-k paradigm (Lacis and Oinas, 1991). $\bar{F}$ represents a broadband flux (solar or thermal infrared; upward or downward) at any vertical level within the AGCM gridcolumn, $F_{n}$ is a similar broadband flux for one of the $N$ subcolumns generated by RRTMG's cloud generator (Räisänen et al., 2004, see below) within the AGCM's gridcolumns, and $f_{n, k}$ is the pseudomonochromatic flux for subcolumn $n$ and spectral point $k$. What the above equation essentially conveys is that a broadband flux which is normally obtained by taking the average over $N$ subcolumns of the sum of $K$ spectral calculations for each subcolumn, is approximated by the sum of $K$ spectral calculations where each spectral point $k$ is paired randomly with one of the $N$ subcolumns, $n_{k}$. Note that when using Eq. (1) the computational cost of the calculation over all subcolumns is the same as that of a full spectral integration of a single (sub)column. The performance of this approximation in large scale models has been tested extensively (e.g., Barker et al., 2008). The main issue of concern is whether the conditional random noise, decreasing as the inverse square root of the number of times Eq. (1) is applied, has any detrimental impact on the simulations. The prior studies and our own tests with GEOS-5 have shown that the McICA noise for sufficiently long runs (at least a month) is of similar magnitude and nature as the internal variability of the model.

An extensive description of the particular cloud generator used in the GEOS-5 implementation of RRTMG is provided by Räisänen et al. (2004). The generator produces subcolumns that have either clear or completely overcast cloud layers. Whether the cloud condensate of a particular layer varies among the subcolumns depends on the assumptions about horizontal cloud heterogeneity, namely either homogeneous or heterogeneous condensate distributions can be specified within the generator. The horizontal location of clouds in a particular layer (i.e., subcolumn assignment) and specific value of condensate (for heterogeneous condensate distributions) depend on cloud presence at other layers according to the overlap rules implemented. By design, in the limit of an infinite number of subcolumns, layer horizontal averages reproduce the vertical profile of cloud fraction and condensate provided as input to the generator by the AGCM. More specific descriptions of rules and assumptions about cloud fraction and condensate distribution overlaps as implemented in GEOS-5 are provided in the section that follows.

\section{Cloud overlap and variability representation}

The cloud fraction overlap options for the cloud generator included in the RRTMG package incorporate the standard assumptions that have been used extensively in the past, i.e., maximum, random, and (the most popular) maximumrandom overlap (Geleyn and Hollingsworth, 1979; Tian and Curry, 1989) where contiguous cloudy layers overlap maximally and randomly otherwise. Räisänen et al. (2004) provides a mathematical description of the practical implementation of these overlap assumptions in a cloud generator 
algorithm. In this work, from the above simplified overlap descriptions, we only test the maximum-random overlap option.

Starting with the work of Hogan and Illingworth (2000), numerous studies (e.g., Mace and Benson-Troth, 2002; Oreopoulos and Khairoutdinov, 2003; Naud et al., 2008) have shown that the above simple overlap assumptions do not capture the vertical structure of cloud fields seen in observations and cloud resolving models, and that the concept of "generalized" cloud fraction overlap represents observed overlap more realistically. In the generalized overlap paradigm, the combined cloud fraction of two cloudy layers at heights $z_{1}$ and $z_{2}$ with separation distance $\Delta z=z_{2}-z_{1}$ can be approximated as a weighted average of combined cloud fractions from maximum and random overlap, $C_{\max }(\Delta z)$ and $\mathrm{C}_{\mathrm{ran}}(\Delta z)$, respectively according to:

$C(\Delta z)=\alpha(\Delta z) C_{\max }(\Delta z)+(1-\alpha(\Delta z)) C_{\operatorname{ran}}(\Delta z)$

where

$C_{\max }(\Delta z)=\max \left(C\left(z_{1}\right), C\left(z_{2}\right)\right)$

$C_{\mathrm{ran}}(\Delta z)=1-\left(1-C\left(z_{1}\right)\right)\left(1-C\left(z_{2}\right)\right)$

The weighting parameter $\alpha(\Delta z)$, is a measure of the proximity of overlap to maximum (exact when $\alpha(\Delta z)=1$ ) or random (exact when $\alpha(\Delta z)=0$ ); Negative values suggest some degree of minimum overlap (a combined cloud fraction greater than that of random overlap). A commonly used simplification, also adopted here, is that $\alpha(\Delta z)$ depends only on the separation distance $\Delta z$ and not on the specific values of $z_{1}$ and $z_{2}$, i.e., cloud fraction overlaps the exact same way at different heights of the atmosphere as long as $\Delta z$ is the same. With this assumption, it was shown (Hogan and Illingworth, 2000) that $\alpha(\Delta z)$ can be fit reasonably well by an inverse exponential function:

$\alpha(\Delta z)=\exp \left(-\frac{\Delta z}{L_{\alpha}}\right)$

where $L_{\alpha}$ is the "decorrelation length" for cloud fraction overlap. Such a fit obviously does not allow for negative values $\alpha(\Delta z)$ which are occassionally observed (e.g., Oreopoulos and Norris 2011). Because the fit provided by Eq. (4) is usually used in conjunction with Eq. (2), generalized overlap has also been termed "exponential-random" overlap (Hogan and Illingworth, 2000).

The manner in which cloud water contents align in the vertical may also be important for processes like radiation (or precipitation). For example, the domain-averaged fluxes differ between a case where all high or low condensate values are aligned to create pockets of vertically integrated high or low water path (WP), and a case where a more random alignment homogenizes the WP horizontal distribution (e.g., see
Norris et al., 2008). The nature of condensate alignment can be expressed in terms of rank correlations of water content as a function of separation distance $\Delta z=z_{2}-z_{1}$ (e.g., see Pincus et al., 2005 and Oreopoulos and Norris 2011). For two layers at heights $z_{1}$ and $z_{2}$ the water contents at both heights can be ranked separately for the overlapping portion of $N_{\text {cld }}$ subcolumns of the two cloud layers. A linear correlation coefficient $r(\Delta z)$ can then be calculated from the ranks $R_{i}\left(z_{1}\right)$ and $R_{i}\left(z_{2}\right)$ according to:

$$
r(\Delta z)=\frac{\sum_{i=1}^{N_{\text {cld }}}\left(R_{i}\left(z_{1}\right)-\bar{R}\left(z_{1}\right)\right)\left(R_{i}\left(z_{2}\right)-\bar{R}\left(z_{2}\right)\right)}{\sqrt{\sum_{i=1}^{N_{\text {cld }}}\left(R_{i}\left(z_{1}\right)-\bar{R}\left(z_{1}\right)\right)^{2}} \sqrt{\sum_{i=1}^{N_{\text {cld }}}\left(R_{i}\left(z_{2}\right)-\bar{R}\left(z_{2}\right)\right)^{2}}}
$$

The rank correlation coefficient expresses the likelihood water contents of the same relative magnitude within their respective layers are aligned in the vertical, with $r\left(z_{1}, z_{2}\right)=1$ corresponding to perfect alignment and $r\left(z_{1}, z_{2}\right)=0$ corresponding to completely random alignment.

It was suggested (e.g.. Räisänen et al., 2004) that the rank correlation coefficient can also be fit by an inverse exponential (which again will not capture the more rarely enncountered negative values) under similar assumptions as for the cloud fraction overlap parameter, i.e., that it is only a function of $\Delta z$ and not $z$ itself

$r(\Delta z)=\exp \left(-\frac{\Delta z}{L_{\mathrm{r}}}\right)$

where $L_{\mathrm{r}}$ is the rank correlation decorrelation length. Large values of $L_{\mathrm{r}}$ indicate condensate values that are highly correlated in terms of relative magnitude, while small values suggest condensate values whose relative magnitude is weakly correlated among layers.

The practical implementation of generalized cloud fraction overlap and condensate overlap using inverse exponential fits is described by Räisänen et al. (2004). The cloud generator that came with RRTMG had generalized cloud fraction overlap capability, but did not allow for overlap of condensate distributions; we added that feature following Räisänen et al. (2004). To create the subcolumns that describe the cloud fields within the GCM gridcolumns, two additional pieces of information, besides the profiles of cloud fraction $C$ and mean condensate (liquid and ice) are needed, namely specification of the decorrelation lengths $L_{\alpha}$ and $L_{\mathrm{r}}$ and of the magnitude of the horizontal variability of the condensate distributions. We defer discussion of decorrelation lengths for the next section, and describe variability here.

To create condensate distributions for cloudy layers we assume that beta distributions describe the horizontal variations of normalized condensate $x=w / w_{\max }$ :

$p_{\beta}(x)=\frac{\Gamma(p+q)}{\Gamma(p) \Gamma(q)} x^{p-1}(1-x)^{q-1}$ 
where $\Gamma$ is the gamma function and the maximum value of condensate $w_{\max }$ is set as five times the assumed variance $\sigma_{w}^{2}$ of the distribution. The shape parameters $p, q$ of the beta distribution are calculated from the method of moments (Wilks, 1995):

$p=\frac{\bar{x}^{2}(1-x)}{\sigma_{x}^{2}}-\bar{x}$

$q=\frac{p(1-\bar{x})}{\bar{x}}$

where $\bar{x}=\bar{w} / w_{\max }$ and $\sigma_{x}^{2}=\sigma_{w}^{2} / w_{\max }^{2}$.

The standard deviation $\sigma_{w}$ of the distribution was set as follows, loosely based on Oreopoulos and Barker (1999) and our own analysis of hydrometeor variability in the CloudSat (Stephens et al., 2002) data:

$$
\begin{array}{ll}
\sigma_{w}=0.5 \bar{w} & \text { when } C>0.99 \\
\sigma_{w}=\bar{w} / \sqrt{2} & \text { when } 0.9 \leq C \leq 0.99 \\
\sigma_{w}=\bar{w} & \text { when } C<0.9
\end{array}
$$

The choice of the beta distribution is supported by observations (Oreopoulos and Davies 1998 and Lee et al., 2010), but other skewed distributions that have also been observed from airborne and satelllite measurements, such as gamma and lognormal would also have been acceptable alternate choices. Equations (7) and (9) apply to both liquid and ice condensate, and in layers where the two phases coexist their ratio is assumed to remain constant across all subcolumns. Since no distinction is made between liquid and ice cloud fraction, the normalized standard deviation $\sigma_{w} / \bar{w}$ is de facto the same for liquid and ice condensate distributions. The beta distribution of normalized condensate $x$ is converted to an actual condensate distribution and then to a cloud optical depth distribution using the AGCM-provided effective particle size which is different for each phase, but assumed horizontally homogeneous. The latter assumption is universal in GCMs, even those equipped with two-moment microphysical schemes. Analysis based on aircraft observations by Räisänen et al. (2003) and modeling results (Barker and Räisänen, 2004) indicate that correlations between WP and effective particle size in liquid clouds can reduce substantially the radiative effects of WP inhomogeneity alone, i.e., optical depth inhomogeneity being weaker than WP inhomogeneity has a notable impact on radiative fluxes. Nonetheless, since the specification of the amount of condensate variability via $\sigma_{w}$ does not come explicitly from the host AGCM or derived from rigorous physical principles, and variability is used only to gauge diagnostically the sensitivity of the cloud radiative effect, we argue that it is not critical to fully justify its exact specification or the specification of optical depth variability itself. Different degrees of variability will have quantitatively different impacts on the cloud radiative effect, but the qualitative impact is nevertheless entirely predictable: larger inhomogeneity results in smaller shortwave
(SW) and longwave (LW) cloud radiative effects at the top of the atmosphere and vice-versa.

\section{Description of AGCM setup and experiments}

\subsection{Specification of overlap parameter decorrelation lengths}

As explained earlier, for the AGCM experiments with generalized cloud fraction overlap and heterogeneous condensate distributions, the decorrelation lengths $L_{\alpha}$ and $L_{\mathrm{r}}$ need to be specified. The simplest option is to select values that are universal, i.e., invariant in space in time. Values that have been used in prior work (Räisänen et al., 2004; Morcrette et al., 2008) are $L_{\alpha}=2 \mathrm{~km}$ and $L_{\mathrm{r}}=1 \mathrm{~km}$. Such a far reaching simplification may not be justifiable in principle on physical grounds given the wide range of cloud regimes. Still, whether a more sophisticated specification of decorrelation lengths is needed in practice should be a matter of further investigation. The availability of cloud particle/hydrometeor reflectivity and backscatter data from the Cloud Profiling Radar (CPR) of the CloudSat mission and the CALIOP lidar of the CALIPSO mission (Winker et al., 2010) potentially allows a more detailed examination of spatiotemporal variation of cloud overlap decorrelation lengths.

We performed such a cloud overlap analysis using CloudSat products for two months, January and July 2009. For cloud fraction overlap we used the 2B-GEOPROF-LIDAR product which provides a cloud mask from combining the different hydrometeor detection capabilities of CPR and CALIOP (CPR is more capable at detecting layers with large concentrations of hydrometeors while CALIOP can better detect unobscured optically thin clouds). For condensate distribution overlap we used CloudSat's 2B-GEOPROF product which provides reflectivities for $\sim 1.7 \mathrm{~km}$ footprints identified to contain hydrometeors at various vertical locations (separated by $\sim 500 \mathrm{~m}$ ). Our rank correlations following Eq. (5) therefore actually come from reflectivities and not cloud condensates which are also available from CloudSat (e.g. product 2B-CWC-RO or 2B-CWC-RVOD), but considered less reliable for the liquid phase due to drizzle and mixed/supercooled clouds often assigned erroneously to the ice phase (Lee et al., 2010). Since reflectivies are proportional to the size of the hydrometeor particles, under the assumption of constant particle number densities, the amount of condensate is monotonically related to particle size and Eq. (5) can be applied to reflectivities as well. A caveat of the 2B-GEOPROF reflectivities on the other hand is that they do not result only from interactions of the radar beam with suspended (cloud) particles, but also precipitation particles. While the above make CloudSat-derived decorrelation lengths approximate, it should be kept in mind that the goal is not to obtain a perfect map of their geographical variation, but to have a plausible broad picture of their spatial and 
seasonal variability that can be contrasted with globally constant decorrelation lengths for cloud radiative effect studies.

Figure 1 shows the zonal distribution of $L_{\alpha}$ (top panel) and $L_{\mathrm{r}}$ (bottom panel) derived via least-square fits (Press et al., 1992) from monthly-averaged CloudSat/CALIPSO $\alpha(\Delta z)$ and $r(\Delta z)$ profiles within $3^{\circ}$ latitude zones, for January and July (solid lines), with the limitations stated earlier. The data segment length used in the above calculation is $100 \mathrm{CPR}$ profiles $(\sim 170 \mathrm{~km})$, similar to the spatial resolution of the AGCM experiments described below. There is a clear zonal structure for both months with tropical latitudes exhibiting larger decorrelation lengths (more maximum overlap and greater vertical alignment of reflectivities of similar relative strength), consistent with documented overlap contrasts between convective and stratiform regimes (Barker, 2008a, b; Oreopoulos and Norris, 2011). $L_{\mathrm{r}}$ values seem to be generally about half those of $L_{\alpha}$, in broad agreement with previous findings (Räisänen et al., 2004; Pincus et al., 2005; Oreopoulos and Norris 2011). Seasonal shifts of the peak values of decorrelation lengths appear to reflect the movement of the Intertropical Convergence Zone (ITCZ).

Our objective for AGCM parameterization purposes is to capture in a simple manner the observed decorrelation length zonal structure shown in Fig. 1. For that purpose, we apply a Gaussian fit (black dashed curves) of the form

$L=m_{1}+m_{2} \exp \left[-\left(\theta-m_{3}\right)^{2} / m_{4}^{2}\right]$

to the January (black) curves. In Eq. (10), $\theta$ is the latitude in degrees and $m_{1}, m_{2}, m_{3}$ and $m_{4}$ are parameter fits. All, except $m_{3}$, are held constant, and their values yielding decorrelation length in $\mathrm{km}$ are provided in Table 1 . Parameter $m_{3}$, controlling the latitude at which Eq. (10) peaks, captures the zonal seasonal movement seen in the CloudSat data, and is allowed to vary as a function of the day of the year according to:

$m_{3}=-4 m_{3,0}(J D-272) / 365$ when $J D>181$

$m_{3}=4 m_{3,0}(J D-91) / 365$ when $J D \leq 181$

where $J D$ is the Julian Day. We set $m_{3,0}=7.0$ (cloud fraction overlap) and $m_{3,0}=8.5$ (condensate/reflectivity overlap). Our approach then in essence consists of assigning the initial Gaussian fit of the monthly-averaged January observations to 1 January, and then finding the zonally-averaged decorrelations for all other days of the year by applying Eqs. (10) and (11). This is how the gray dashed curves in Fig. 1 (for 1 July) were obtained. Note that the January fits describe the zonal distribution of both decorrelation lengths more realistically than the July curves which are not fits to the data, but outcomes of the parameterization expressed by Eqs. (10) and (11). The parameterized northward shift of the January curves intended to capture July overlap generally leads to underestimates. Again, for the purposes of this study, where
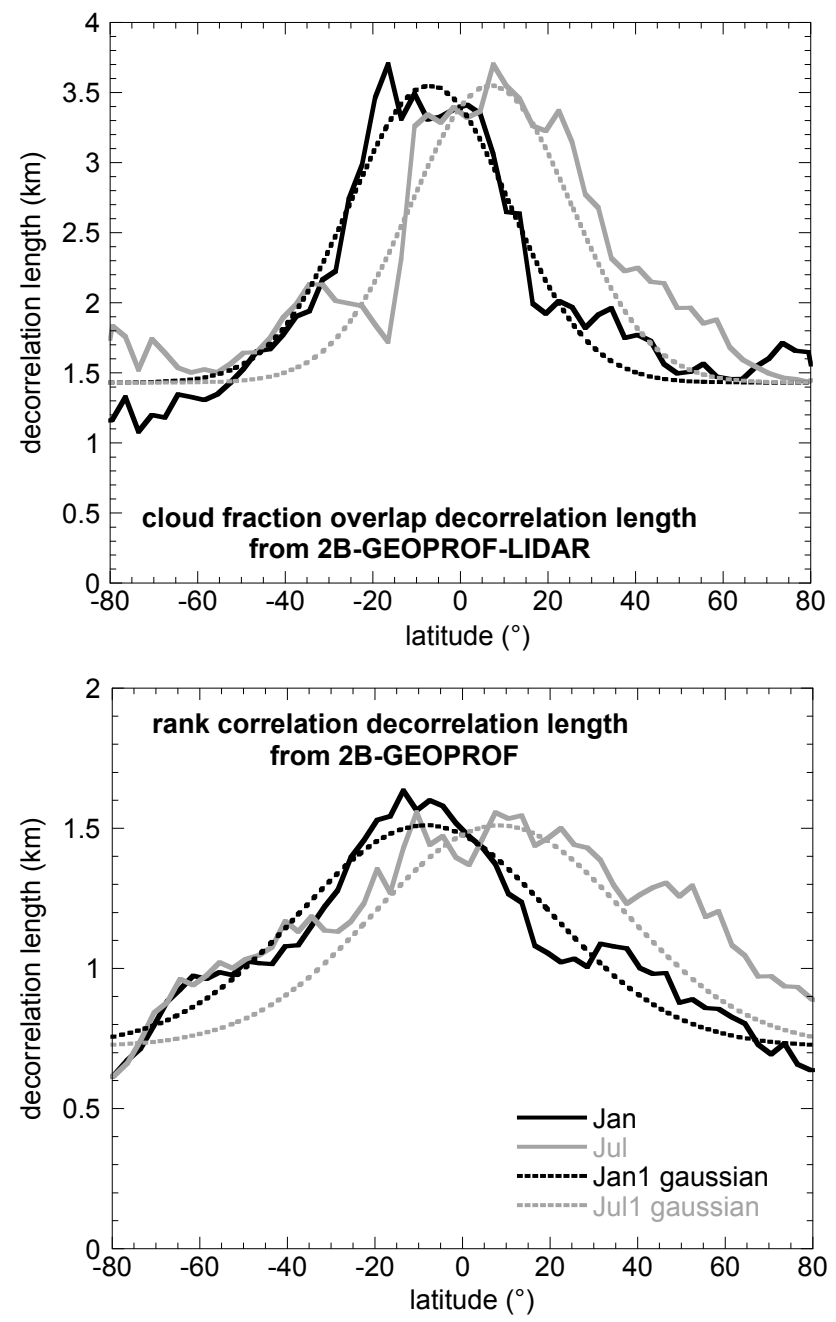

Fig. 1. (top): Cloud fraction overlap decorrelation lengths from $3^{\circ}$ zonal averages of $\alpha(\Delta z)$ for January and July 2009 (solid curves) derived from the 2B-GEOPROF-LIDAR CloudSat product; the dashed curves correspond to gaussian fits according to Eqs. (10) and (11). (bottom): As top panel, but for rank correlation decorrelation lengths calculated from CloudSat 2B-GEOPROF CPR reflectivities.

the goal is to examine the sensitivity of the cloud radiative effect to a range of decorrelation length specifications and the differences arising when the exact same overlap assumptions are applied to two different cloud schemes, the imperfect matching to observed overlap (itself coming with its own limitations) is acceptable.

\subsection{Description of AGCM experiments with diagnostic radiation}

To examine the changes in the radiative impact of clouds when different assumptions are invoked about (a) the horizontal heterogeneity of their condensate; (b) the way their condensate distributions overlap; and (c) the way their cloud fractions overlap, relatively short $(\sim 1 \mathrm{yr}$ simulations with the 
Table 1. Parameters for the Gaussian fits per Eqs. (10) and (11) of zonal decorrelation lengths shown in Fig. 1.

\begin{tabular}{lll}
\hline $\begin{array}{l}\text { Fit parameters } \\
\text { for Eqs. (10)-(11) }\end{array}$ & $\begin{array}{l}\text { Cloud fraction } \\
\text { overlap }\end{array}$ & $\begin{array}{l}\text { Condensate } \\
\text { overlap }\end{array}$ \\
\hline$m_{1}$ & 1.43 & 0.72 \\
$m_{2}$ & 2.12 & 0.79 \\
$m_{3,0}$ & -7.00 & -8.50 \\
$m_{4}$ & -25.58 & 40.40 \\
\hline
\end{tabular}

GEOS-5 AGCM are conducted with the RRTMG radiation package producing "diagnostic" only fluxes. Had we wanted to examine the full impact of our cloud changes on the model climate much longer simulations of at least a decade with interactive RRTMG would have been necessary. By diagnostic RRTMG radiation fields we mean that the heating and cooling rates produced by RRTMG are not supplied back to the AGCM to affect dynamical and physical processes. Instead, the model run is driven by the radiation fields produced by the original (operational) radiation package (Chou and Suarez 1999; Chou et al., 2001) which treats clouds according to its default configuration, as usual. The McICA version of RRTMG simply runs side-by-side with the original radiation package and operates on the cloud fields produced by the standard model, but as transformed by the cloud generator in accordance with our heterogeneity and overlap assumptions.

Our suite of experiments is summarized in Table 2. All experiments were run with the GEOS-5 AGCM Fortuna v. 2.5 at $2 \times 2.5^{\circ}$ resolution with 72 vertical levels, and differ only in their assumptions about cloud fields. While all experiments share the same profiles of cloud fraction and mean condensate, other assumptions about the nature of the clouds are different from experiment to experiment. Clouds can be assumed to be horizontally homogeneous or heterogeneous and their cloud fractions can overlap according to either the maximum-random or generalized overlap paradigms. When clouds are heterogeneous and overlap according to the maximum-random overlap assumption, a condensate decorrelation length still needs to be supplied. All simulations correspond to 13-month runs from which the last 12 months are considered for analysis; prescribed sea surface temperatures for the period May 1993 to May 1994 are used.

Two sets of experiments were conducted. One where the standard (control) cloud scheme (Molod et al., 2012) operates, and one with McRAS-AC (Sud et al., 2012; Sud and Lee, 2007). The two cloud schemes share the same convective scheme (Relaxed Arakawa-Schubert or RAS), but with different assumptions about the onset of convection, and ambient air entrainment (quadratic in McRAS versus linear in standard RAS) and are substantially different in their stratiform cloud parameterizations and microphysics descriptions. The control cloud scheme has pre-specified liquid and ice particle sizes, while McRAS-AC has active two-
Table 2. List of experiments conducted with the GEOS-5 AGCM running two different cloud schemes to assess the effects of cloud heterogeneity and overlap on the cloud radiative effect.

\begin{tabular}{|c|c|}
\hline Experiment ID & Description \\
\hline 1 & $\begin{array}{l}\text { Homogeneous clouds, maximum-random } \\
\text { overlap }\end{array}$ \\
\hline 2 & $\begin{array}{l}\text { Heterogeneous clouds (Eq. 9), maximum- } \\
\text { random overlap, } L_{\mathrm{r}}=1 \mathrm{~km}\end{array}$ \\
\hline 3 & $\begin{array}{l}\text { Homogeneous clouds, generalized overlap, } \\
L_{\alpha}=2 \mathrm{~km}\end{array}$ \\
\hline 4 & $\begin{array}{l}\text { Heterogeneous clouds, generalized over- } \\
\text { lap, } L_{\alpha}=2 \mathrm{~km}, L_{\mathrm{r}}=1 \mathrm{~km}\end{array}$ \\
\hline 5 & $\begin{array}{l}\text { As Exp. 4, but with the standard deviation } \\
\text { of Eq. (9) halved }\end{array}$ \\
\hline 6 & As Exp. 4 , but with $L_{\alpha}=4 \mathrm{~km}, L_{\mathrm{r}}=2 \mathrm{~km}$ \\
\hline 7 & As Exp. 4 , but with $L_{\alpha}=2 \mathrm{~km}, L_{\mathrm{r}}=2 \mathrm{~km}$ \\
\hline 8 & $\begin{array}{l}\text { Heterogeneous clouds, generalized overlap } \\
\text { from CloudSat/CALIPSO }\end{array}$ \\
\hline
\end{tabular}

moment cloud microphysics where condensate amounts, particle sizes, and precipitation depend on the aerosol loading. For our experiments we chose to provide McRAS-AC with a present day climatology of aerosol mass concentrations produced by the GOCART (Chin et al., 2000) chemical transport model. Note that for both sets of experiments, while the aerosols are radiatively active in the operational radiation package that provides interactive radiation fields, they are not accounted for by RRTMG which produces the diagnostic radiation fields used to assess overlap radiative impacts on CRE.

For each of the experiments we generate the monthly, seasonal and annual geographical distribution of the LW and $\mathrm{SW}$ cloud radiative effect (CRE) at the top of the atmosphere (TOA). The CRE is defined as:

$\mathrm{CRE}_{\mathrm{LW}, \mathrm{SW}}=F_{\mathrm{LW}, \mathrm{SW}}^{\mathrm{clr}}-F_{\mathrm{LW}, \mathrm{SW}}^{\mathrm{all}}$

which can also be written as

$$
\mathrm{CRE}_{\mathrm{LW}, \mathrm{SW}}=C_{\mathrm{tot}}\left(F_{\mathrm{LW}, \mathrm{SW}}^{\mathrm{clr}}-F_{\mathrm{LW}, \mathrm{SW}}^{\mathrm{ovc}}\right)
$$

where $F$ is the outgoing flux (LW or SW) at the TOA, clr designates clear (cloudless) skies, all a mixture of clear and cloudy skies, and ovc overcast skies (100\% cloud fraction); $C_{\text {tot }}$ is the total vertically projected cloud fraction. The modeled CRE always comes from Eq. (12a); nevertheless, Eq. (12b) which applies when the all-sky flux is written as the linear combination of clear and overcast fluxes, can be used for interpreting the CRE, since a gridcolumn's $C_{\text {tot }}$ is not uniquely defined, but rather depends on the cloud fraction overlap assumption (for the same cloud fraction profile, the closer the overlap to random, the larger $C_{\text {tot }}{ }^{1}$ ). For the

\footnotetext{
${ }^{1}$ Minimum overlap of various degrees produces even larger $C_{\mathrm{tot}}$, but there is no such overlap in our experiments.
} 


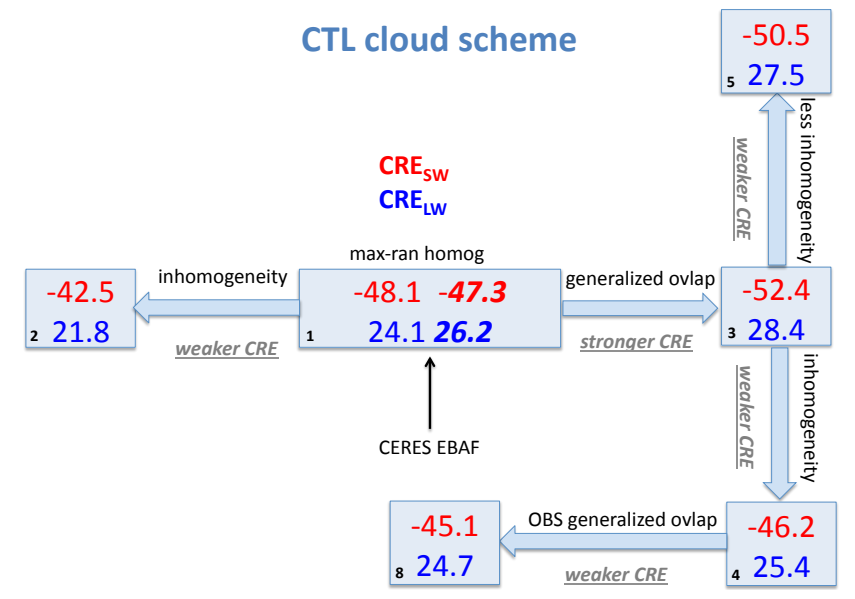

Fig. 2. Box chart providing diagnostic $\mathrm{CRE}$ in $\mathrm{Wm}^{-2}$ (blue for $\mathrm{CRE}_{\mathrm{LW}}$, red for $\mathrm{CRE}_{\mathrm{SW}}$ ) for GEOS-5 CTL scheme experiments with the RRTMG radiation package where cloud condensate distributions change from homogeneous to heterogeneous and overlap changes from maximum-random to generalized. The numbers in italics in the center box are observed values from the CERES EBAF data set. The numbers in the left bottom corner of the boxes are the experiment IDs according to Table 2.

complete intercomparison of CRE among all experiments we use globally-averaged values. For select experiments we also compare zonal (latitudinal) averages and geographical distributions. Although not critical for understanding the sensitivity of CRE to cloud heterogeneity and overlap, we also include in our comparison TOA CRE from the CERES EBAF v. 2.6 data set (Loeb et al., 2009) for the period March 2000 to June 2011.

\section{Analysis of Cloud Radiative Effect dependencies}

\subsection{Global changes in CRE}

We first focus on the sensitivity of globally-averaged CRE to different assumptions about how to generate cloud fields from profiles of cloud fraction and mean condensate. Figures 2 and 3 chart this sensitivity for the control (CTL) and McRAS-AC cloud schemes, respectively. The center box contains AGCM results for the "default" (reference) configuration, namely homogeneous condensate distributions and maximum-random cloud fraction overlap (Exp. 1, see Table 2). Blue numbers depict $C_{R E}$ and red $C R E_{S W}$ values. This box also contains the observed global CREs according to the CERES EBAF (Loeb et al., 2009) product. The other boxes show the various global CRE magnitudes for different assumptions about the nature of the cloud fields.

For the CTL cloud scheme (Fig. 2) when cloud fraction overlap remains maximum-random, but clouds are allowed to be inhomogeneous according to Eqs. (7)-(9) (leftmost box, corresponding to Exp. 2), $\mathrm{CRE}_{\mathrm{LW}}$ decreases by

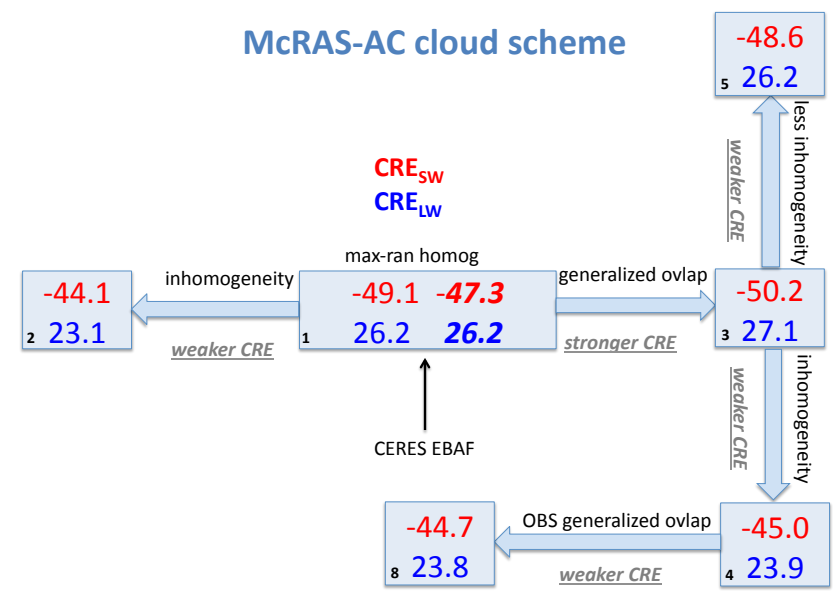

Fig. 3. As Fig. 3, but when McRAS-AC has replaced the GEOS-5 control cloud scheme.

$2.3 \mathrm{Wm}^{-2}\left(21.8 \mathrm{Wm}^{-2}\right)$ and $\mathrm{CRE}_{\mathrm{SW}}$ also decreases in absolute value (i.e., a smaller negative value) by $5.6 \mathrm{Wm}^{-2}$ $\left(-42.5 \mathrm{Wm}^{-2}\right)$. This is because for the same mean condensate, heterogeneous clouds reflect less solar radiation (e.g., Cahalan et al., 1994) and emit less (transmit more) LW radiation (Barker and Wielicki, 1997). For this particular case therefore changes in CRE can be attributed to changes in $F_{\mathrm{LW}, \mathrm{SW}}^{\mathrm{ovc}}$ in Eq. (12b): the SW outgoing flux for overcast conditions is reduced, while the LW outgoing flux increases; in both cases the contrast with the clear-sky flux is reduced. The change in $\mathrm{CRE}_{\mathrm{SW}}$ is more than double that on $\mathrm{CRE}_{\mathrm{LW}}$ since the nonlinearity of the LW emittance curve is restricted to a much narrower range of cloud condensates (or, strictly speaking, optical depths) than the nonlinearity of the SW albedo curve. In other words, changes in the details of an optical depth distribution begin to matter less (because of saturation in emittance) at lower values of mean cloud optical depth. When clouds remain homogeneous, on the other hand, but the cloud fraction overlap changes to generalized (with globally constant $L_{\alpha}=2 \mathrm{~km}$, Exp. 3), it is $C_{\text {tot }}$ in Eq. (12b) that is mainly affected (it appears from our results that the change in the distribution of cloud tops exposed to space, which matters for the $\mathrm{LW}$, is a lesser contributor) both $\mathrm{CRE}_{\mathrm{LW}}$ and $\mathrm{CRE}_{\mathrm{SW}}$ increase by $4.3 \mathrm{Wm}^{-2}$ (to 28.4 and $-52.4 \mathrm{Wm}^{-2}$, respectively; box 3 ), indicating that for the CTL cloud scheme $C_{\text {tot }}$ for generalized overlap is higher than that for maximum-random overlap.

When condensate heterogeneity is applied under conditions of generalized overlap (Exp. 4, lower right box), the effect of increased $C_{\text {tot }}$ in the CTL cloud scheme is entirely eliminated for $\mathrm{CRE}_{\mathrm{SW}}$ by the decrease in $F_{\mathrm{SW}}^{\mathrm{Ovc}}$, but only partially cancelled out for $\mathrm{CRE}_{\mathrm{LW}}$ through increase in $F_{\mathrm{LW}}^{\mathrm{ovc}}$. The end result is that $\mathrm{CRE}_{\mathrm{SW}}$ is weaker by $1.9 \mathrm{Wm}^{-2}$ compared to the reference Exp. 1, while $\mathrm{CRE}_{\mathrm{LW}}$ remains stronger, but by only $1.3 \mathrm{Wm}^{-2}$. Note that the effect of inhomogeneity on CRE is stronger when cloud fraction obeys 
generalized overlap (from Exp. 3 to Exp. 4) than when it obeys maximum-random overlap (from Exp. 1 to Exp. 2): in the former case $\mathrm{CRE}_{\mathrm{SW}}$ and $\mathrm{CRE}_{\mathrm{LW}}$ decrease in strength by $6.2 \mathrm{Wm}^{-2}$ and $3 \mathrm{Wm}^{-2}$, respectively, while for the latter case they decrease by $5.6 \mathrm{Wm}^{-2}$ and $2.3 \mathrm{Wm}^{-2}$. When the standard deviation used for the beta distribution of condensate is halved compared to Eq. (9) (box 5), CRE $\mathrm{SW}_{\mathrm{Sw}}$ is reduced by about $2 \mathrm{Wm}^{-2}$, while $\mathrm{CRE}_{\mathrm{LW}}$ is reduced by $1 \mathrm{Wm}^{-2}$ reaffirming again the fact that any changes that affect overcast fluxes rather than cloud fractions have greater impact in CTL on the SW compared to the LW.

A simultaneous change in both cloud fraction and condensate overlap can be achieved by switching from globally constant decorrelation lengths to CloudSat-based decorrelation lengths (Eqs. 10-11 and Fig. 1). This process is represented by the transition from Exp. 4 to Exp. 8 shown by the bottom two boxes (4 and 8) of Fig. 2. CRE strength decreases by $1.1 \mathrm{Wm}^{-2}$, while $\mathrm{CRE}_{\mathrm{LW}}$ decreases by $0.7 \mathrm{Wm}^{-2}$. One can see that transitioning from homogeneous maximum-random overlap to inhomogeneous clouds following a CloudSat-based generalized overlap results ultimately in $3 \mathrm{Wm}^{-2}$ weaker CRE $\mathrm{SW}_{\mathrm{SW}}$ than Exp. 1, but a slightly stronger (by $0.6 \mathrm{Wm}^{-2}$ ) $\mathrm{CRE}_{\mathrm{LW}}$. This is possible because while cloud fraction changes (from maximum-random to generalized) have about the same effect on both the SW and LW CRE, overcast flux changes (from condensate overlap and inhomogeneity) are too weak in the LW to reverse the increased CRE resulting from generalized overlap.

The CRE response to condensate heterogeneity and generalized overlap when imposed on the cloud fields of an alternate cloud scheme can be substantially different than the one discussed above. This is shown in Fig. 3, which is the same as Fig. 2, but for the McRAS-AC cloud scheme. Cloud water inhomogeneity under conditions of maximumrandom cloud fraction overlap (box 2) results in a slightly smaller weakening of $C E_{S W}$, and a slightly greater weakening of $C R E_{L W}$. This is likely because of the generally optically thinner clouds of the McRAS-AC scheme. The transition of homogeneous clouds from maximum-random overlap to generalized overlap (box 3) gives a much smaller CRE response for McRAS-AC $\left(\sim 1 \mathrm{Wm}^{-2}\right.$ compared to $\sim 4 \mathrm{Wm}^{-2}$ for CTL). Adding inhomogeneity to clouds obeying generalized overlap has about the same CRE effect for McRAS$\mathrm{AC}$ as adding inhomogeneity to clouds following maximumrandom overlap (CRE changes from Exp. 3 to Exp. 4 are about the same as the changes from Exp. 1 to Exp. 2); for the CTL cloud scheme the CRE impacts diverged by $0.6-0.7 \mathrm{Wm}^{-2}$ ). The box corresponding to Exp. 5 indicates that when the imposed inhomogeneity is reduced by half on clouds following generalized overlap, the outcome is close to the reference CRE values, i.e., the effects of modified overlap and inhomogeneity largely cancel out; this was not the case for the CTL cloud scheme for which overlap had a much stronger CRE impact than reduced inhomogeneity. Finally, the change from globally constant decorrelation lengths to

\section{Impact of decorrelation length scale}

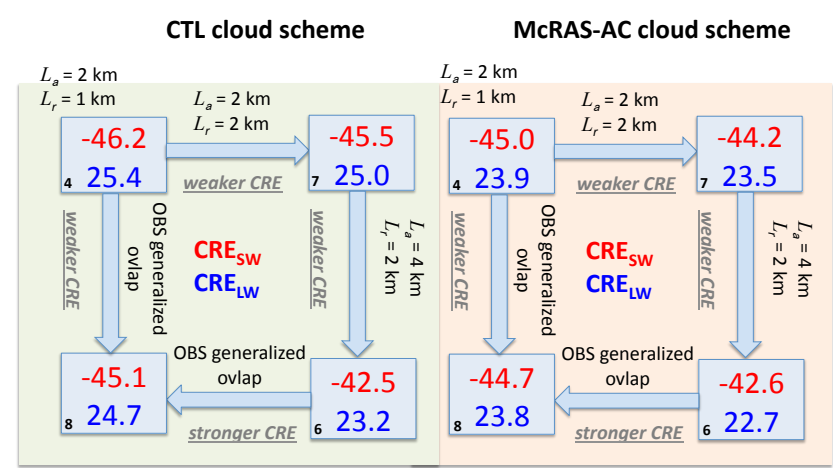

Fig. 4. CRE magnitudes for various decorrelation length values in the generalized overlap paradigm for the CTL cloud scheme (left) and the McRAS-AC cloud scheme (right). The numbers in the left bottom corner of the boxes are the experiment IDs according to Table 2 .

zonally-dependent decorrelation lengths (Exp. 4 to Exp. 8) is notably smaller for the McRAS-AC cloud fields compared to the CTL cloud scheme.

This latter result is also included in Fig. 4 which focuses on CRE changes brought by changing the parameters (i.e., decorrelation lengths) of generalized overlap. The left part of the figure provides global CRE impacts for the CTL cloud scheme while the right part of the figure does the same for the McRAS-AC scheme. In this figure the reference CREs come from Exp. 4 (heterogeneous clouds, generalized overlap with constant decorrelation lengths), upper left box (box 4). The transition from Exp. 4 to Exp. 7 (top boxes 4 and 7) captures the effect of changing the condensate overlap decorrelation length $L_{\mathrm{r}}$. When it is doubled from 1 to $2 \mathrm{~km}$ in the CTL simulations both $\mathrm{CRE}_{\mathrm{SW}}$ and $\mathrm{CRE}_{\mathrm{LW}}$ decrease in strength slightly. This is the result of more aligned condensate distributions increasing the variability in integrated WP compared to shorter $L_{\mathrm{r}}$ (more random overlap of layer condensate distributions producing more homogeneous WP distributions) and consequently yielding reduced $\mathrm{TOA} F_{\mathrm{SW}}^{\mathrm{ovc}}$ and increased $F_{\mathrm{LW}}^{\mathrm{ovc}}$, and thus smaller contrast with the upwelling clear sky flux. If the global decorrelation length of cloud fraction $L_{\alpha}$ is doubled from 2 to $4 \mathrm{~km}$ (transition from Exp. 7 to Exp. 6, right boxes) the reduced $C_{\text {tot }}$ of the less random overlap yields further reductions of $3 \mathrm{Wm}^{-2}$ and $1.8 \mathrm{Wm}^{-2}$ in $\mathrm{CRE}_{\mathrm{SW}}$ and $\mathrm{CRE}_{\mathrm{LW}}$, respectively. Such greater impact of cloud fraction overlap changes compared to condensate distribution overlap changes was also shown by Barker and Räisänen (2005). Because the observed decorrelation lengths are generally smaller than those of Exp. 6, when they are applied in the cloud generator (transition from Exp. 6 to Exp. 8, bottom boxes) the CREs increase again (higher $C_{\text {tot }}$ and more homogeneous distributions of WP) and become comparable to those of Exp. 7. For the CTL cloud scheme, the overall impact of using CloudSat-based decorrelation lengths instead of 


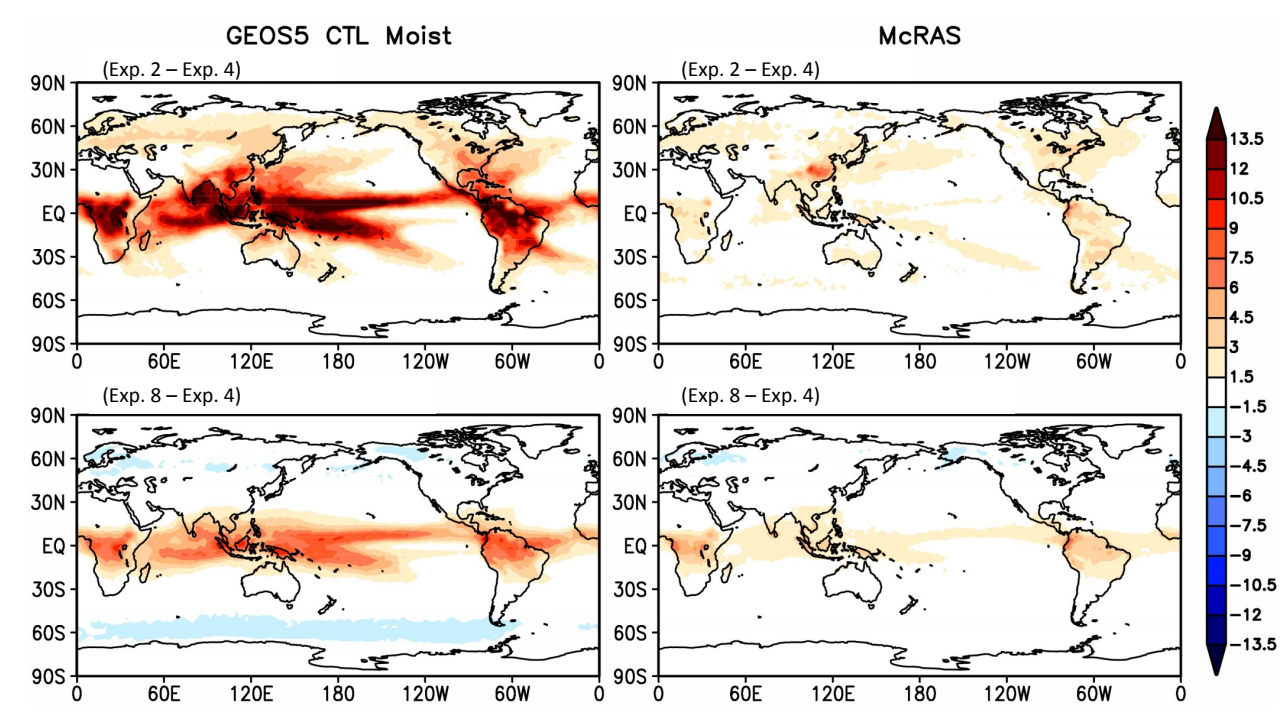

Fig. 5. Maps of annually averaged CRE $\mathrm{SW}$ differences between the Exp. 2 and Exp. 4 (top) and between Exp. 8 and Exp. 4 (bottom). The left panels are for the CTL cloud scheme, while the right panels are for McRAS-AC.

the previously used global values of $L_{\alpha}=2 \mathrm{~km}$ and $L_{\mathrm{r}}=1 \mathrm{~km}$ (Exp. 4 to Exp. 8., left boxes) is about $1 \mathrm{Wm}^{-2}$, slightly more for $\mathrm{CRE}_{\mathrm{SW}}$ and slightly less for $\mathrm{CRE}_{\mathrm{LW}}$. These differences are at first glance rather small to justify the effort of deriving zonally-dependent decorrelation lengths, especially since the Exp. 4 CREs of CTL are already below CERES EBAF and the more sophisticated treatment of overlap makes the discrepancy from observed CREs worse. But as will be shown below, the rather benign global CRE changes conceal local impacts that are much more substantial.

The right part of Fig. 4 contains the exact same analysis as the left part, but for the McRAS-AC scheme implemented in GEOS-5. The impact of doubling the rank correlation decorrelation length (Exp. 4 to Exp. 7) is about the same as for CTL, but doubling the overlap decorrelation length does not change CRE as much for McRAS-AC. The Exp. 4 and Exp. 6 to Exp. 8 transitions are also weaker in terms of CRE changes for McRAS-AC. When these results are considered in conjunction with Fig. 3, the obvious conclusion is that McRASAC cloud distributions do not cause as big CRE changes as those of CTL in response to the different prescriptions of cloud overlap. We attempt to explain why this is the case in Sect. 5.3.

As a concluding thought for this part of the analysis we would like to point out that if $\mathrm{CRE}_{\mathrm{SW}}$ is overestimated and $\mathrm{CRE}_{\mathrm{LW}}$ underestimated compared to observations, as is the case for the CTL cloud scheme, it is not possible to bring both closer to observations through changes in inhomogeneity and overlap descriptions alone. Inhomogeneity reduces $\mathrm{CRE}_{\mathrm{SW}}$ and can bring model and observations closer, but it also reduces the already too low $\mathrm{CRE}_{\mathrm{LW}}$. Similarly, increasing $\mathrm{CRE}_{\mathrm{LW}}$ via changes in overlap (i.e., increasing $C_{\mathrm{tot}}$ ) to match observations has the undesired effect of making the
$\mathrm{CRE}_{\mathrm{SW}}$ overestimates worse. To match both components of CRE to observations, inhomogeneity and overlap changes must be accompanied by concurrent changes in other cloud properties such as cloud top height and mean condensate.

\subsection{Geographical changes in CRE}

In this subsection we examine whether the relatively narrow range of global CRE impact due to changes in cloud overlap specification conceals a much wider range of regional CRE changes. For the sake of brevity, we focus on only two overlap specification changes, the transition from maximumrandom overlap to generalized overlap with globally constant decorrelation lengths (with heterogeneous clouds), and the transition from the latter type of overlap to generalized overlap with zonally variable decorrelation lengths as parameterized per the CloudSat data analysis. In other words we examine regional CRE differences between Exp. 2 and Exp. 4 and between Exp. 8 and Exp. 4.

Figure 5 shows maps of annually averaged $\mathrm{CRE}_{\mathrm{SW}}$ differences between the experiments mentioned above, while Fig. 6 is a counterpart figure for $\mathrm{CRE}_{\mathrm{LW}}$. The panels in the top row correspond to Exp. 2 minus Exp. 4 differences, and the panels in the bottom row to Exp. 8 minus Exp. 4 differences; the left panels are for the CTL cloud scheme and the right panels for McRAS-AC. The CTL cloud scheme yields substantially greater CRE differences for the transition from maximum-random to generalized overlap than between two generalized overlaps, and in the tropics compared to midlatitudes. Zonal CRE differences between Exp. 2 and Exp. 4 peak at $\sim 11 \mathrm{Wm}^{-2}$ in the SW and $\sim-10 \mathrm{Wm}^{-2}$ in the $\mathrm{LW}$ around $5^{\circ} \mathrm{N}$ (left panels of Fig. 7) reflecting changes in $C_{\text {tot }}$ of $\sim 0.13$ (blue curve in the top panel of Fig. 8). The counterpart CRE differences between Exp. 8 and Exp. 4 

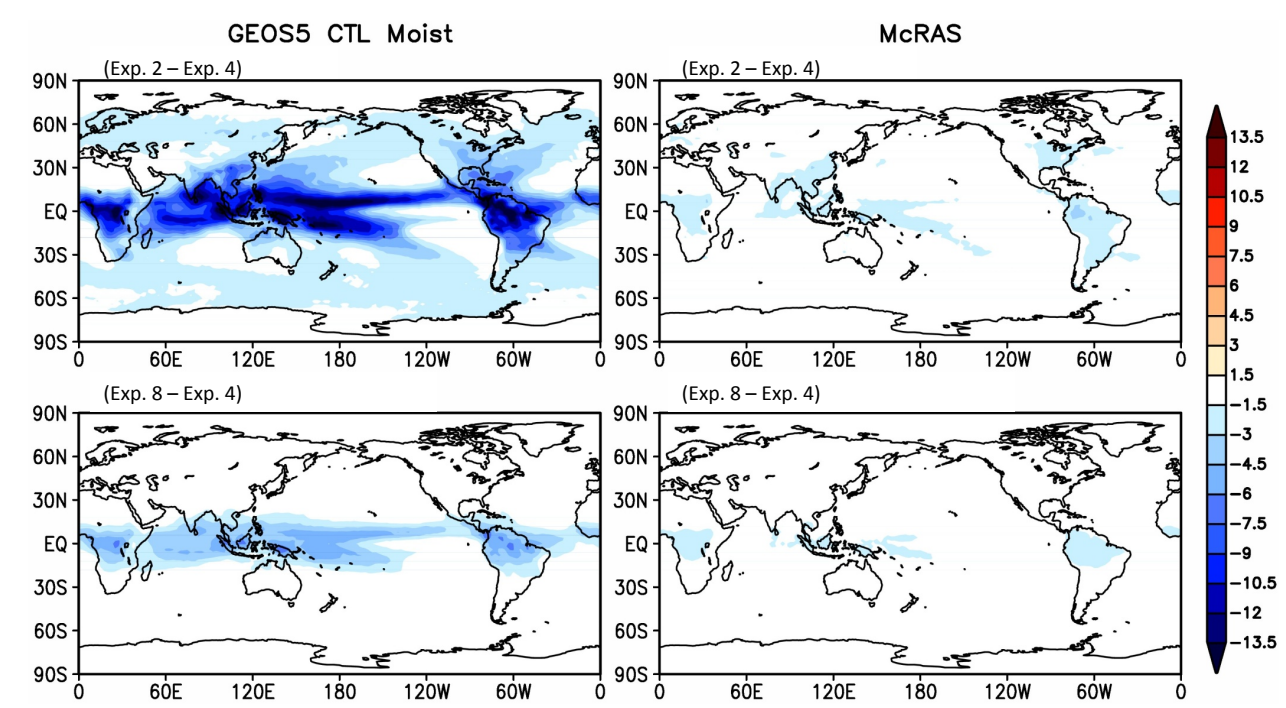

Fig. 6. As Fig. 5, but for $\mathrm{CRE}_{\mathrm{LW}}$.
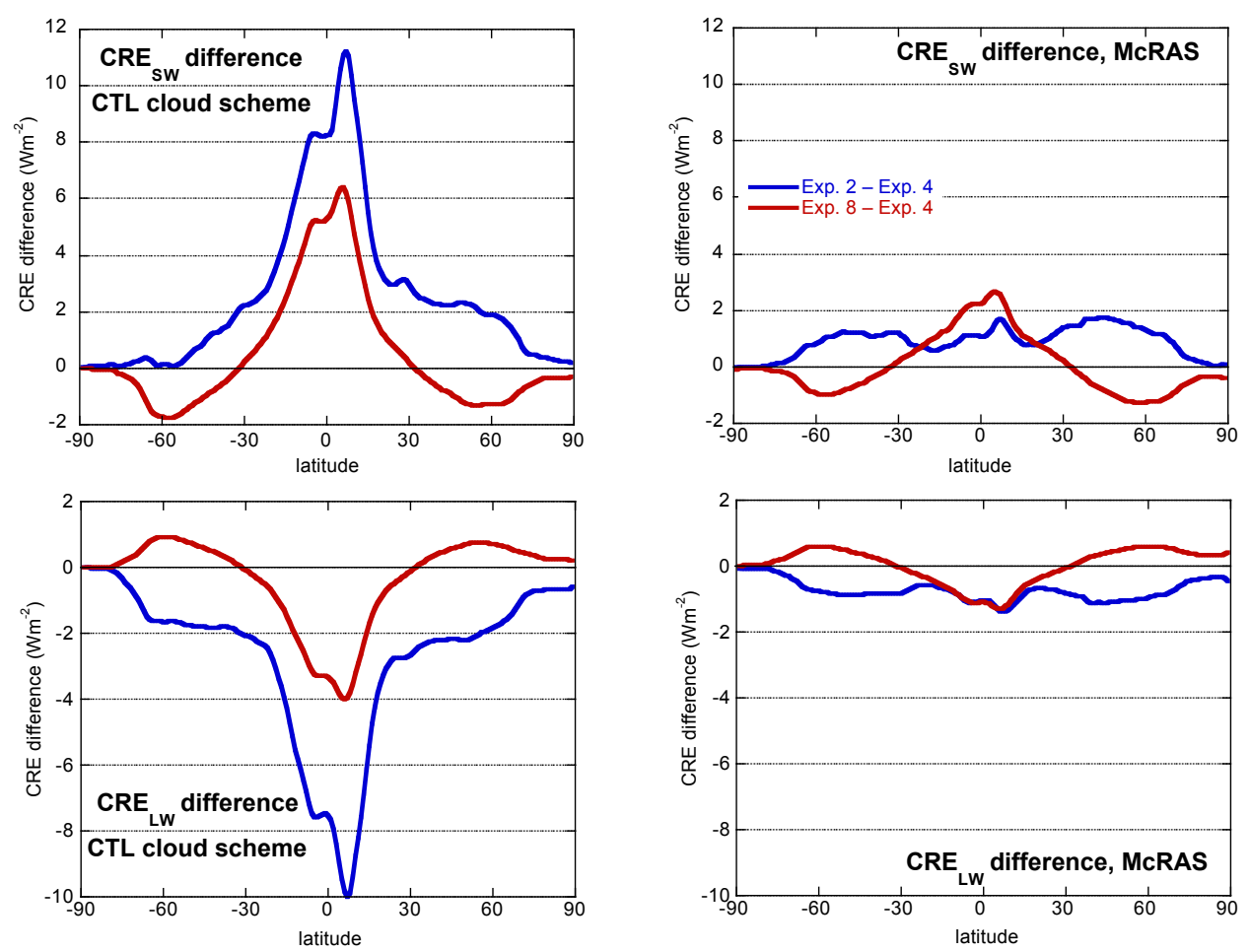

Fig. 7. Zonal averages of the differences shown in Figs. 5 and 6. The left panels are for the CTL cloud scheme, the right panels are for McRAS-AC. Top panels are for $\mathrm{CRE}_{\mathrm{SW}}$, while the bottom panels are for $\mathrm{CRE}_{\mathrm{LW}}$.

are $\sim 6 \mathrm{Wm}^{-2}$ and $\sim-4 \mathrm{Wm}^{-2}$ for a $C_{\text {tot }}$ change of about 0.05 (red curve in the top panel of Fig. 8); in this case however the different vertical alignment of condensate distributions also contributes to the CRE differences, making the $\mathrm{CRE}_{\mathrm{SW}}$ and $\mathrm{CRE}_{\mathrm{LW}}$ changes more distinct. It is interesting that the sign of the CRE differences between Exp. 8 and Exp. 4 (changes in the details of generalized overlap) is not the same everywhere. While the $\mathrm{CRE}_{\mathrm{SW}}\left(\mathrm{CRE}_{\mathrm{LW}}\right)$ difference is generally positive (negative), at midlatitudes there are negative (positive) differences with peaks at about 60 degrees latitude. The difference in behaviour from tropics to midlatitudes is solely due the parameterization of the CloudSat-based decorrelation lengths in Fig. 1. The constant decorrelation lengths are lower than those from CloudSat 

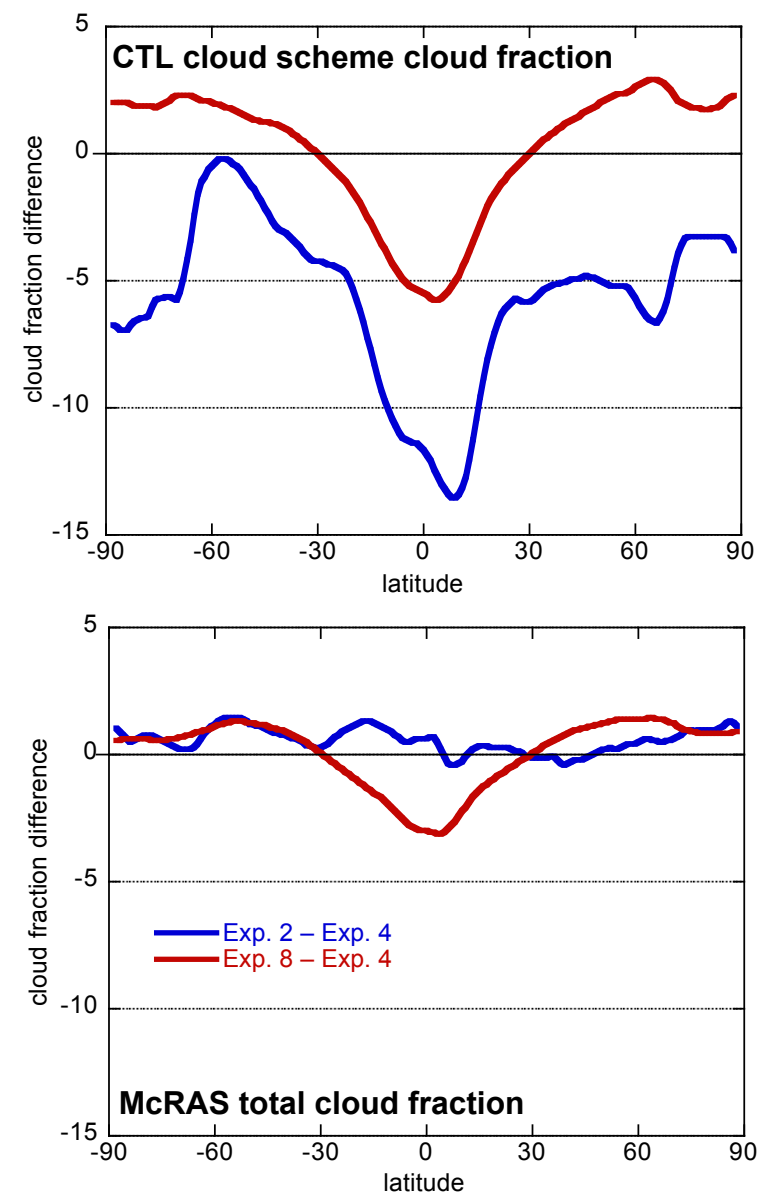

Fig. 8. Zonally-averaged differences of $C_{\text {tot }}$ (on a scale 0-100) for Exp. 2-Exp. 4 (blue curves) and Exp. 8-Exp. 4 (red curves). The top panel is for the CTL cloud scheme, while the bottom panel is for McRAS-AC.

in the tropics and yield higher $C_{\text {tot }}$ and less variable WPs, ergo, stronger CRE (expressed as positive $\mathrm{CRE}_{\mathrm{SW}}$ and negative $\mathrm{CRE}_{\mathrm{LW}}$ differences). In the midlatitudes, the opposite is true, i.e., the globally constant values are higher than the CloudSat-based parameterized decorrelation lengths resulting in weaker CREs for Exp. 4 compared to Exp. 8 (negative $\mathrm{CRE}_{\mathrm{SW}}$ and positive $\mathrm{CRE}_{\mathrm{LW}}$ differences).

The counterpart McRAS-AC CRE differences are much weaker, as can be seen in the right panels of Figs. 5, 6, and 7, consistent with much smaller changes in $C_{\text {tot }}$ (Fig. 8) and the smaller global CRE differences noted earlier in Figs. 3 and 4. The zonal structure of the Exp. 8 minus Exp. 4 CRE differences can be explained by invoking the same arguments as before for the CTL cloud scheme, but exhibit notably smaller values. The Exp. 2 minus Exp. 4 CRE differences also have the same sign as in CTL across all latitudes, but exhibit a much weaker latitudinal dependence with no tropical peak as in CTL, while being also substantially smaller. One interesting feature seen in the bottom panel of Fig. 8 is that the zonally-averaged $C_{\text {tot }}$ difference of Exp. 2 minus
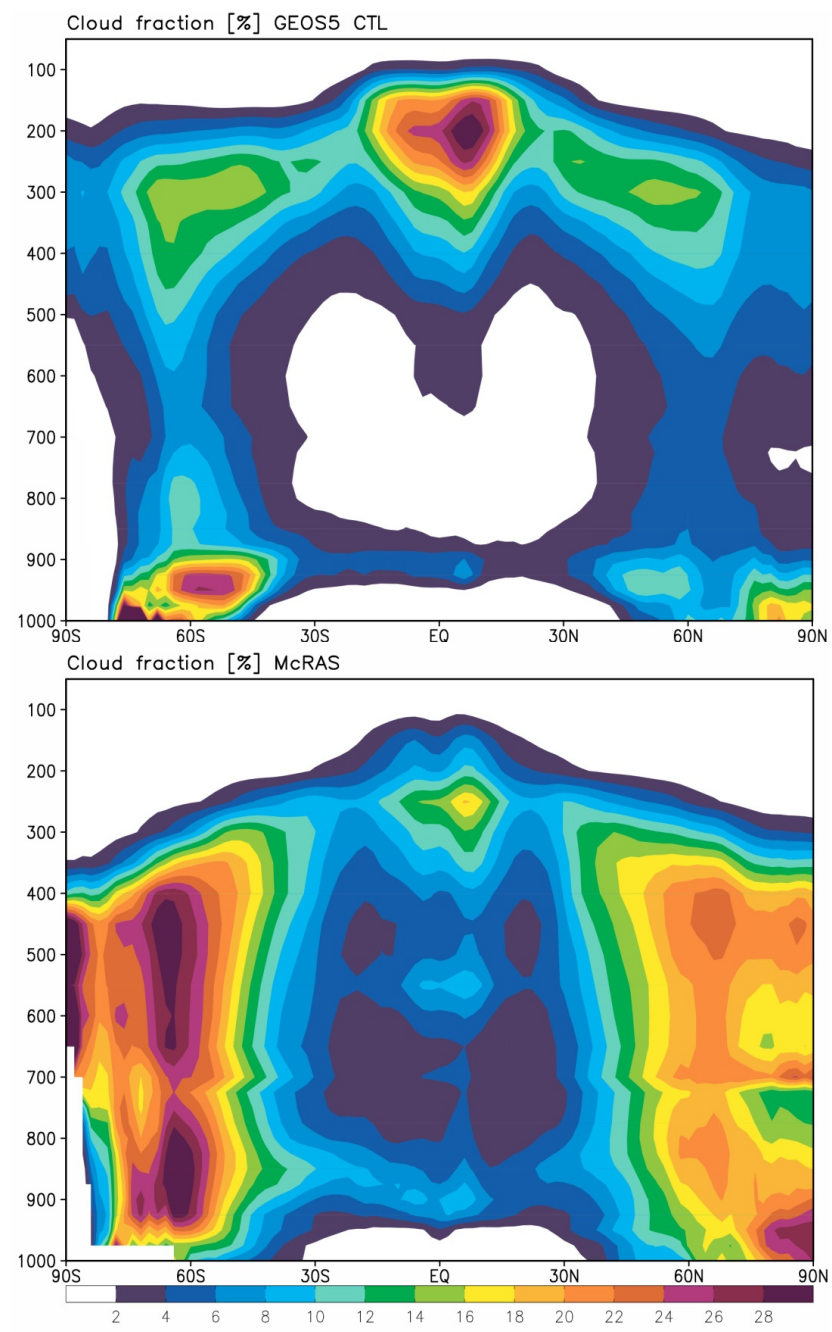

Fig. 9. Annually- and zonally-averaged cloud fraction profiles (on a scale 0-100) for the CTL and McRAS-AC cloud schemes.

Exp. 4 is small and generally positive, in contrast to CTL. This means that there are many instances where $C_{\text {tot }}$ from maximum-random overlap exceeds that of generalized overlap, but not in a way that will create larger overall CREs. This in turn points to cloud vertical profiles in McRAS-AC where the random part (cloudy layers separated by clear layers) of maximum-random overlap is invoked more often than in CTL. Recall that within the realm of generalized overlap, exact random cloud fraction overlap can only occur in the limit of an infinite decorrelation length.

\subsection{Why overlap details in the two cloud schemes affect CRE differently}

The quite distinct CRE response of the two cloud schemes when the cloud generator is furnished with identical rules to produce cloudy subcolumns from common profiles of cloud fraction and mean condensate for radiation 


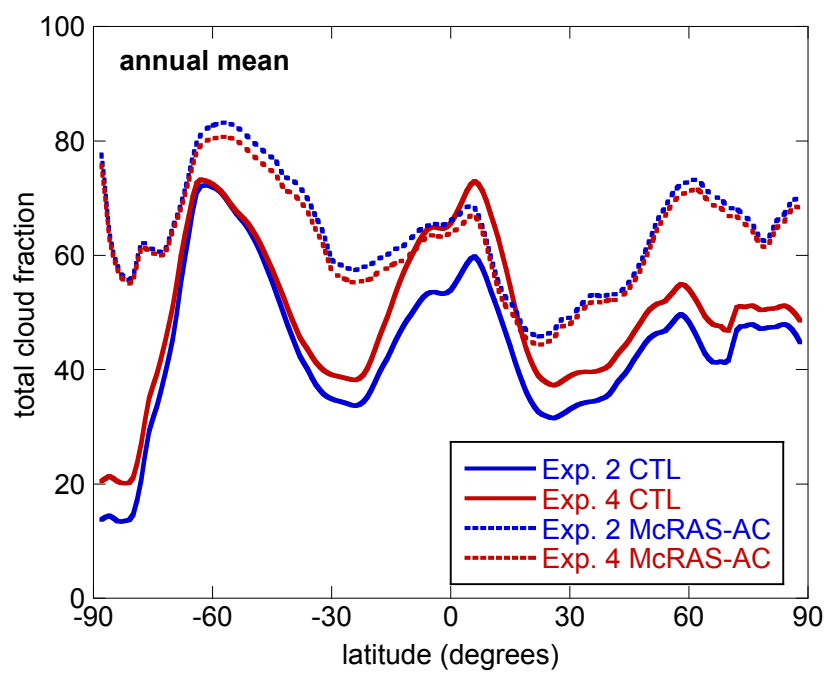

Fig. 10. Annually- and zonally-averaged total cloud fraction $C_{\text {tot }}$ (on a scale 0-100) for Exp. 2 and Exp. 4 cloud fraction overlap assumptions applied to CTL and McRAS-AC cloud schemes.

calculations, merits further examination. Since the largest impact comes from the overlap of cloud fraction, we examine here how the two schemes differ in terms of cloud fraction means and distributions, and the frequency of multilayer cloud occurrences.

First we examine the one-year cloud fraction climatology produced by the two schemes. We compare in Fig. 9 annually- and zonally-averaged cloud fraction profiles produced by CTL (top) and McRAS-AC (bottom). The differences between the two panels are striking. McRAS-AC produces in general larger cloud fractions throughout the entire extent of the midlatitude and polar troposphere and the largest part of the tropical troposphere. The CTL cloud scheme on the other hand produces higher cloud fractions at the upper levels of the tropical troposphere due to deep convection, and exhibits some cloud presence at the higher altitudes of the midlatitude atmosphere where McRAS-AC produces no clouds. The eventual outcome of these average cloud fraction profiles is that $C_{\text {tot }}$ is higher for the McRASAC cloud scheme. This is clearly demonstrated in the Fig. 10 zonal plot showing $C_{\text {tot }}$ from Exp. 2 (maximum-random overlap) and Exp. 4 (generalized overlap with $L_{\alpha}=2 \mathrm{~km}$ ). The figure makes apparent that McRAS-AC produces higher zonal cloud fractions everywhere for Exp. 2 and nearly everywhere (except a portion of the tropics) for Exp. 4. The higher cloud fractions for McRAS-AC come with much greater insensitivity to the overlap specification (the distance between the blue and red dashed curves, also shown as difference in Fig. 8). Indeed, larger cloud fractions make the details of overlap more inconsequential since the difference between maximum, random and any degree in between (i.e., generalized), becomes smaller at the high end of the cloud fraction distribution.

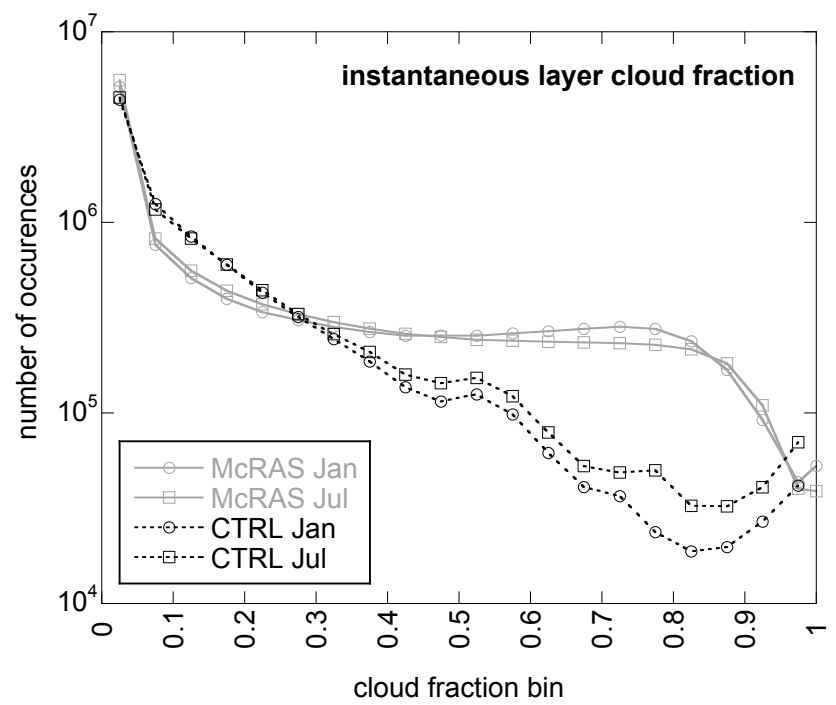

Fig. 11. Frequency distributions of twice-daily sampled instantaneous layer cloud fraction during January and July within the period of our runs. The cloud fraction bins are 0.05 wide, with a separate bin for completely overcast conditions. The first bin does not include clear skies.

A better way to demonstrate the tendency of McRASAC to produce higher cloud fractions is to examine instantaneous layer cloud fractions. We produced distributions for this quantity for both cloud schemes from twice-daily samples extracted during January and July within the period of our runs. The four distributions are shown in Fig. 11. The seasonal differences are not pronounced, especially for McRAS$\mathrm{AC}$, but the differences between the two cloud schemes is remarkable. McRAS-AC generates many more layer cloud fractions in the 0.5-0.9 range, and also produces overcast cloud layers which the CTL scheme never does. The smaller zonal averages of total cloud fraction by the CTL cloud scheme in Fig. 10 appear therefore to be the outcome of consistently lower than McRAS-AC occurrences of instantaneous layer cloud fractions above 0.5 .

Another factor making the details of overlap specification matter less is the number of cloudy layer within a gridcolumn at a particular instance. The more layers are simultaneously cloudy in a model gridcolumn, the greater the chance that they will be farther apart, and therefore the greater the tendency towards random overlap conditions either under maximum-random overlap or generalized overlap. In this regard, McRAS-AC is again distinct from CTL in producing more occurrences of larger numbers of model layers being simultaneously cloudy (Fig. 12) at a particular instance.

All the above results portray a consistent picture: McRAS$\mathrm{AC}$ is more cloudy than CTL under a variety of metrics and high cloud fractions are produced with greater frequency so that the exact overlap specification is less consequential on $C_{\text {tot }}$ and CRE. 


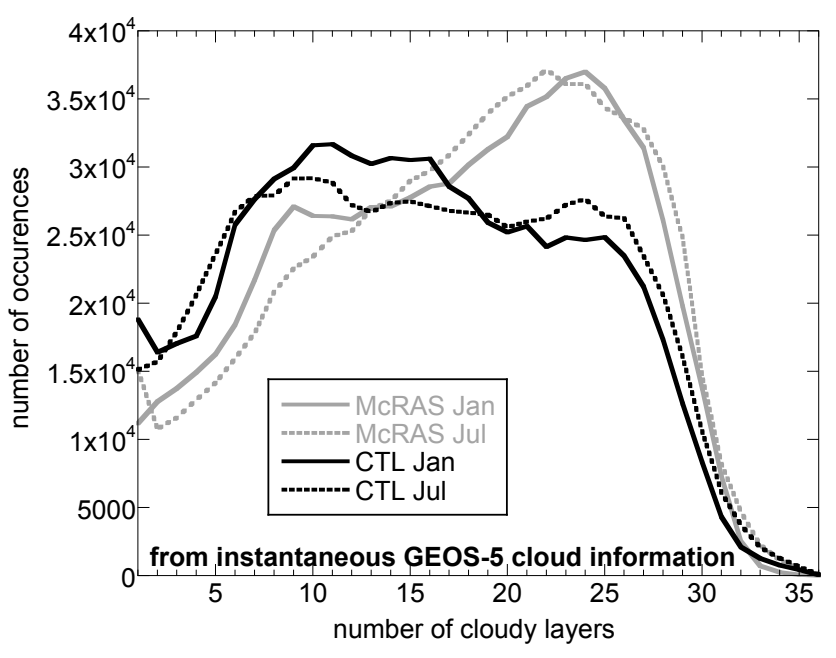

Fig. 12. Frequency distributions of instantaneous multi-layer cloud occurences using the same data as in Fig. 11.

\section{Discussion and conclusions}

While earlier studies have shown that vertical cloud structure and particularly cloud fraction overlap can have large instantaneous effects, especially on solar fluxes (Barker et al., 1999), global effects within climate models have not been as systematically quantified. New capabilities in describing arbitrary cloud fraction and condensate overlaps within GCMs that resemble more faithfully the vertical cloud structures observed in nature, along with progress on how radiation schemes handle these more complex cloud fields, has been improving the current state of affairs. Our study was stimulated by this progress and sought to address the following question: Do the details of cloud overlap matter radiatively to a similar extent when applied exactly the same way on the (different) mean cloud fraction and condensate fields produced by two distinct cloud schemes? We found the answer to be negative. One cloud scheme's cloud distributions change the radiative fluxes much more than the other's after overlap was manipulated. Therefore, no conclusive answer on whether the details of cloud vertical structure matter much for radiation can be given: it will depend on the host model and/or its cloud scheme. In contrast, the influence of cloud condensate heterogeneity may indeed be more consistent across cloud schemes, and the same is likely to be true for the vertical overlap of inhomogeneous condensate distributions which appears to have only a small impact.

The radiative consequences of cloud vertical structure and condensate heterogeneity were studied in this paper diagnostically, in other words, changes in radiation brought about by these factors did not feed back into the model. In that sense, our study resembles that of Shonk and Hogan (2010) who examined the radiative impact of different assumptions about condensate horizontal variability and cloud overlap as implemented on cloud fields from re-analysis data. In that study the global effects of cloud fraction overlap (their "vertical shift") on SW and LW CRE were (absolute values) $\sim 4 \mathrm{Wm}^{-2}$ and $\sim 2 \mathrm{Wm}^{-2}$. The experiment transition from which these numbers were obtained are roughly equivalent to our transitions from Exp. 2 to Exp. 4 (see Figs. 2 and 3). In our case the change in CRE is $\sim 3.6 \mathrm{Wm}^{-2}$ for both the $\mathrm{SW}$ and LW in the CTL cloud scheme; the alternate McRAS-AC cloud scheme produces CRE changes slightly below $1 \mathrm{Wm}^{-2}$. Hence, that studies of this type may eventually put an upper limit on the global impact of cloud overlap in current large scale models, but with a range of outcomes that may remain quite wide. Even greater variability range is expected to occur at smaller spatial scales. Our zonal average peak CRE impact is $\sim 10 \mathrm{Wm}^{-2}$, for both SW and LW CRE while that of Shonk and Hogan (2010) reaches such values (with much less zonal structure) only in the SW; the LW peak is about half, consistent with their global result.

We did not discuss much the level of agreement of simulated CRE for our different experiments with observed CRE. This was a conscious decision since agreement, at global levels at least, can be achieved through appropriate tuning of various cloud properties. Figures 2 and 3 show that the best agreement is not necessarily achieved with the most realistic assumptions about the nature of cloud field structure. Nevertheless, it should be noted that if one of the CRE components is overestimated and the other underestimated, both cannot be simultaneously pushed towards observations by adjusting cloud condensate heterogeneity and overlap assumptions alone. This is because any change that strengthens one component of CRE will have the undesired effect of acting likewise on the other component as well.

Acknowledgements. The authors gratefully acknowledge support by the NASA Modeling Analysis and Prediction and CloudSat/CALIPSO Science Team Recompete programs managed by David Considine. Computational resources and support were provided from the NASA Center for Climate Simulation (NCCS). We would also like to thank M. Iacono and E. Mlawer of AER for their assistance in implementing RRTMG into GEOS-5.

Edited by: B. Mayer

\section{References}

Barker, H. W.: Overlap of fractional cloud for radiation calculations in GCMs: A global analysis using CloudSat and CALIPSO data, J. Geophys. Res., 113, D00A01, doi:10.1029/2007JD009677, 2008 a.

Barker, H. W.: Representing cloud overlap with an effective decorrelation length: An assessment using CloudSat and CALIPSO data, J. Geophys. Res., 113, D24205, doi:10.1029/2008JD010391, 2008b.

Barker, H. W. and Wielicki, B. A.: Parameterizing grid-averaged longwave fluxes for inhomogeneous marine boundary layer clouds, J. Atmos. Sci., 54, 2785-2798, 1997. 
Barker, H. W. and Räisänen, P.: Neglect by GCMs of subgrid-scale horizontal variations in cloud droplet effective radius: A diagnostic radiative analysis, Q. J. Roy. Meteor. Soc., 130, 1905-1920, 2004.

Barker, H. W. and Räisänen, P.: Radiative sensitivities for cloud structural properties that are unresolved by conventional GCMs, Q. J. Roy. Meteor. Soc., 131, 3103-3122, 2005.

Barker, H. W., Stephens, G. L., and Fu, Q.: The sensitivity of domain-averaged solar fluxes to assumptions about cloud geometry, Q. J. Roy. Meteor. Soc., 125, 2127-2152, 1999.

Barker, H. W., Cole, J. N. S., Morcrette, J.-J., Pincus, R., Räisänen, P., von Salzen, K., Vaillancourt, P. A.: The Monte Carlo Independent Column Approximation: An assessment using several global atmospheric models, Q. J. Roy. Meteor. Soc., 134, 14631478, 2008.

Chin, M., Rood R. B., Lin S.-J., Muller J.-F., and Thompson, A. M.: Atmospheric sulfur cycle simulated in the global model GOCART: Model description and global properties. J. Geophys. Res., 105, 24671-24687, 2000.

Chou, M.-D. and Suarez M. J.: A solar radiation parameterization for atmospheric studies, Technical Report Series on Global Modeling and Data Assimilation, NASA/TM-1999-10460, 15, 52 pp., 1999.

Chou, M.-D., Suarez M. J., Liang X.-Z., and Yan, M. H.: A thermal infrared radiation parameterization for atmospheric studies, Technical Report Series on Global Modeling and Data Assimilation, NASA/TM-2001-104606, 19, 65 pp., 2001.

Clough, S. A., Shephard, M. W., Mlawer, E. J., Delamere, J. S., Iacono, M. J., Cady-Pereira, K., Boukabara, S., and Brown, P. D.: Atmospheric radiative transfer modeling: a summary of the AER codes, J. Q. Spectrosc. Radiat. Transf., 91, 233-244, 2005.

Geleyn, J. F. and Hollingsworth, A.: An economical analytical method for the computation of the interaction between scattering and line absorption of radiation, Contrib. Atmos. Phys., 52, 1-16, 1979.

Hogan, R. J. and Illingworth, A. J.: Deriving cloud overlap statistics from radar, Q. J. R. Meteor. Soc., 126, 2903-2909, 2000.

Hogan, R. J. and Illingworth, A. J.: Parameterizing ice cloud inhomogeneity and the overlap of inhomogeneities using cloud radar data, J. Atmos. Sci., 60, 756-767, 2003.

Iacono, M. J., Delamere, J. S., Mlawer, E. J., Shephard, M. W., Clough, S. A., and Collins, W. D: Radiative forcing by long-lived greenhouse gases: Calculations with the AER radiative transfer models, J. Geophys. Res., 113, D13103, doi:10.1029/2008JD009944, 2008.

Lacis, A. A. and Oinas, V.: A description of the correlated k distribution method for modeling nongray gaseous absorption, thermal emission, and multiple scattering in vertically inhomogeneous atmospheres, J. Geophys. Res., 96, 9027-9063, 1991.

Lee, S., Kahn, B. H., and Teixeira, J.: Characterization of cloud liquid water content distributions from CloudSat, J. Geophys. Res., 115, D20203, doi:10.1029/2009JD013272, 2010.

Loeb, N. G., Wielicki, B., Doelling, D., Smith, G., Keyes, D., Kato, S., Manalo-Smith, N., and Wong, T.: Toward optimal closure of the earth's top-of-atmosphere radiation budget, J. Climate, 22, 748-766, 2009.

Mace, G. G. and Benson-Troth, S.: Cloud layer overlap characteristics derived from long-term cloud radar data, J. Climate, 15, 2505-2515, 2002.
Mlawer, E. J., Taubman, S. J., Brown, P. D., Iacono, M. J., and Clough, S. A.: RRTM, a validated correlated-k model for the longwave, J. Geophys. Res., 102, 16663-16682, 1997.

Molod, A., Takacs, L., Suarez, M. J., Bacmeister, J., Song, I.-S., Eichmann, A., and Chang, Y.: The GEOS-5 atmospheric General Circulation Model: mean climate and development from MERRA to Fortuna. NASA, Technical Report Series on Global Modeling and Data Assimilation, NASA/TM-2008-104606, 28, 112 pp., 2012.

Morcrette, J. J., Barker, H. W. Cole, J. N. S., Iacono, M. J., and Pincus, R.: Impact of a new radiation package, McRad, in the ECMWF Integrated Forecasting System. Mon. Weather Rev., 136, 4773-4798, 2008.

Naud, C. M., Del Genio, A., Mace, G. G., Benson, S., Clothiaux, E. E., and Kollias, P.: Impact of dynamics and atmospheric state on cloud vertical overlap, J. Climate, 21, 17581770, doi:10.1175/2007JCLI1828.1, 2008.

Norris, P. M., Oreopoulos L., Hou, A. Y., Tao, W. K., and Zeng, X.: Representation of 3D heterogeneous cloud fields using copulas: Theory for water clouds, Q. J. Roy. Meteorol. Soc., 134, 18431864, 2008.

Oreopoulos L. and Davies R.: Plane Parallel Albedo Biases from Satellite Observations. Part II: Parameterizations for Bias Removal, J. Climate, 11, 933-944, 1998.

Oreopoulos L. and Barker H. W.: Accounting or subgrid-scale cloud variability in a multi-layer $1 \mathrm{D}$ solar radiative transfer algorithm, Q. J. Roy. Meteor. Soc., 125, 301-330, 1999.

Oreopoulos, L. and Khairoutdinov, M.: Overlap properties of clouds generated by a cloud-resolving model, J. Geophys. Res. 108, 4479, doi:10.1029/2002JD003329, 2003.

Oreopoulos, L. and Norris, P. M.: An analysis of cloud overlap at a midlatitude atmospheric observation facility, Atmos. Chem. Phys., 11, 5557-5567, doi:10.5194/acp-11-5557-2011, 2011.

Pincus, R., Barker, H. W., and Morcrette, J. J: A fast, flexible, approximate technique for computing radiative transfer in inhomogeneous cloud fields, J. Geophys. Res., 108, 4376, doi:10.1029/2002JD003322, 2003.

Pincus, R., Hannay, C., Klein, S. A., Xu, K.-M., and Hemler, R.: Overlap assumptions for assumed probability distribution function cloud schemes in large-scale models, J. Geophys. Res., 110, D15S09, doi:10.1029/2004jd005100, 2005.

Press, W. H., Teukolsky, S. A., Vetterling, W. T., and Flannery, B. P.: Numerical recipes in Fortran 77, the art of scientific computing, 2nd ed., Cambridge University Press, 933 pp., 1992.

Räisänen, P., Isaac G. A., Barker, H. W., and Gultepe, I.: Solar radiative transfer for stratiform clouds with horizontal variations in liquid water path and droplet effective radius, Q. J. Roy. Meteor. Soc., 129, 2135-2149, 2003.

Räisänen, P., Barker, H. W., Khairoutdinov M., Li, J., and Randall, D. A.: Stochastic generation of subgrid-scale cloudy columns for large-scale models, Q. J. Roy. Meteor. Soc., 130, 2047-2067, 2004.

Rienecker M. M, Suarez M. J., Todling R., Bacmeister J., Takacs L., Liu H.-C., Gu W., Sienkiewicz M., Koster, R. D., Gelaro R., Stajner I., and Nielsen J. E.: The GEOS-5 Data Assimilation System - Documentation of Versions 5.0.1, 5.1.0, and 5.2.0. NASA/TM2008-104606, . 27, 118 pp., 2008.

Shonk, J. K. P. and Hogan R. J.: Effect of improving representation of horizontal and vertical cloud structure on the earth's global 
radiation budget. Part II: the global effects, Q. J. Roy. Meteor. Soc., 136, 1205-1215, doi:10.1002/qj.646, 2010.

Stephens, G. L., Vane, D. G., Boain, R. J., Mace, G. G., Sassen, K., Wang, Z., Illingworth, A. J., O’Connor, E. J., Rossow, W. B., Durden, S. L., Miller, S. D., Austin, R. T., Benedetti, A., Mitrescu, C., and CloudSat Science Team: A new dimension of space-based observations of clouds and precipitation, B. Am. Meteor. Soc., 83, 1771-1790, 2002.

Sud, Y. C. and Walker, G. K.: Microphysics of Clouds with the Relaxed Arakawa-Schubert Scheme (McRAS), Part I: Design and Evaluation with GATE Phase III Data, J. Atmos. Sci., 56, 31963220, 1999.

Sud, Y. C. and Lee, D.: Parameterization of aerosol indirect effect to complement McRAS cloud scheme and its evaluation with the 3year ARM-SGP analyzed data for single column models, Atmos. Res., 86, 105-125, 2007.

Sud, Y. C., Lee, D., Oreopoulos, L., Barahona, D., Nenes, A., and Suarez, M. J.: Performance of McRAS-AC in the GEOS5 AGCM: aerosol-cloud-microphysics, precipitation, cloud radiative effects, and circulation, Geosci. Model Dev. Discuss., 5, 1381-1434, doi:10.5194/gmdd-5-1381-2012, 2012.
Tian, L. and Curry, J. A.: Cloud overlap statistics, J. Geophys. Res., 94, 9925-9935, 1989.

Tiedtke, M.: An extension of cloud-radiation parameterization in the ECMWF model: The representation of sub-grid scale variations of optical depth, Mon. Weather Rev., 124, 745-750, 1996.

Wilks, D. S.: Statistical methods in the atmospheric sciences, Academic Press, 464 pp., 1995.

Winker, D. M., Pelon, J., Coakley, J. A., Jr., Ackerman, S. A., Charlson, R. J., Colarco, P. R., Flamant, P., Fu, Q., Hoff, R. M., Kittaka, C., Kubar, T. L., Le Treut, H., McCormick, M. P., Mégie, G., Poole, L., Powell, K., Trepte, C., Vaughan, M. A., and Wielicki, B. A.: The CALIPSO Mission: A Global 3D View of Aerosols and Clouds, B. Am. Meteor. Soc., 91, 1211-1229, 2010. 